EPJ Web of Conferences 59, 09005 (2013)

DOI: $10.1051 /$ epjconf/20135909005

(C) Owned by the authors, published by EDP Sciences, 2013

\title{
Longitudinal bunch compression study with induction voltage modulator
}

\author{
Akira Nakayama ${ }^{1, a}$, Yasuo Sakai ${ }^{1}$, Yoshifumi Miyazaki ${ }^{1}$, Takashi Kikuchi ${ }^{2}$, \\ Mitsuo Nakajima ${ }^{1}$ and Kazuhiko Horioka ${ }^{1}$ \\ ${ }^{1}$ Department of Energy Sciences, Tokyo Institute of Technology, Nagatsuta 4259, Midori-ku, \\ Yokohama 226-8502, Japan \\ ${ }^{2}$ Department of Electrical Engineering, Nagaoka University of Technology, 1603-1 \\ Kamitomioka, Niigata 940-2188, Japan
}

\begin{abstract}
For the beam driver of inertial confinement fusion, the technology to compress a charged particle beam in longitudinal direction is crucially important. However, the quality of the beam is expected to be deteriorated when the beam is rapidly compressed in longitudinal direction. In order to investigate the beam dynamics during bunch compression, we made a compact beam compression system and carried out beam compression experiments. In this paper, we show the background of our study and recent progress of the beam compression experiments.
\end{abstract}

\section{INTRODUCTION}

According to the concept of heavy ion fusion, the beam is accelerated with long pulse and low current during the low energy domain, and the beam current increases by longitudinal compression as beam energy increases $[1,2]$. The beam current is expected to increase about 10 times at the final stage of the accelerator. However, the space charge effect may increase the beam emittance and disturb the compression rate during the bunch compression [3]. Emittance is an index to express the quality of beam and is defined by the volume in 6 dimensional phase space, and the beam cannot get the expected compression ratio when emittance increases. As factors to decrease the beam compression ratio, following factors are considered [4, 5]: (1) accuracy of the compression voltage waveform, (2) initial energy spread of charged particles, (3) space charge effect, and (4) collective effects induced by non-stationary space charge field. Above all factors, our final goal is to analyze the collective effect on the emittance growth. When the beam bunch is modulated adiabatically, emittance can keep a constant value. However, it is not the case in the final stage of the high power accelerators. We are going to make the bunch compression experiments with abrupt bunch compression using induction voltage modulator. The modulated beam is compressed through a solenoidal transport line. All of the emittance increase can be reflected only to the longitudinal direction by limiting the influence of traverse motion of the beam. In other words, the beam current waveform observed in the experiment includes all influence factors for compression ratio. Basically, we can include the factors (1)-(3) in numerical calculations. We can evaluate the collective effect on the emittance growth by comparing experimental value with the numerical calculation in which factors (1)-(3) are included. This time, we focus on factor (2): initial energy spread of charged particles.

\footnotetext{
ae-mail: khorioka@es.titech.ac.jp
}

This is an Open Access article distributed under the terms of the Creative Commons Attribution License 2.0, which permits unrestricted use, distribution, and reproduction in any medium, provided the original work is properly cited. 


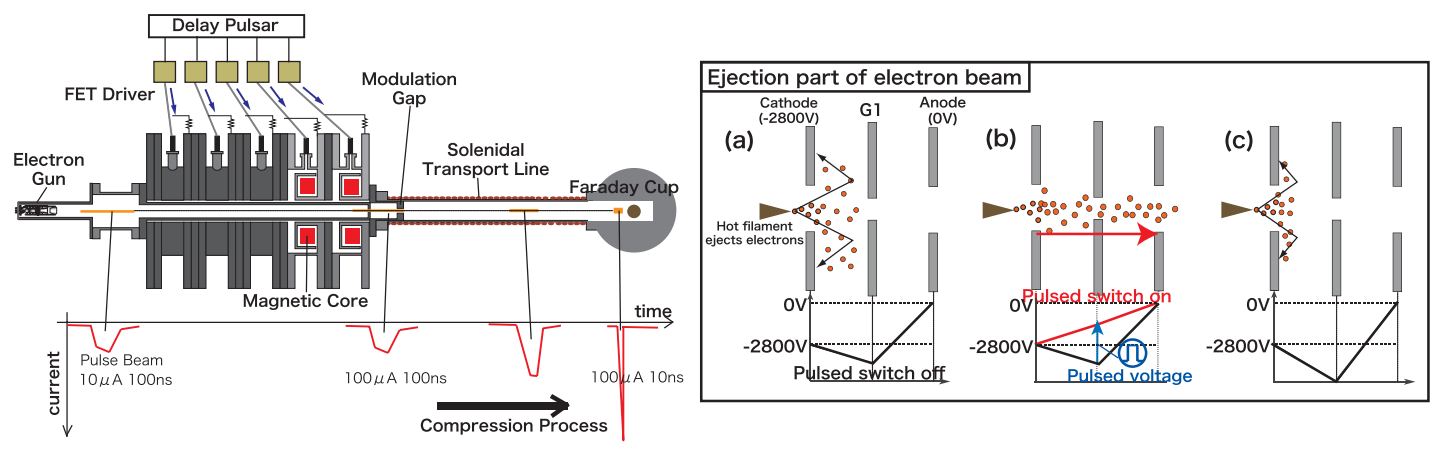

Figure 1. Left figure: setup for beam compression experiment. Right figure: schematic illustration of electron behavior in gated electron beam source (a) For gate off, (b) For gate on, (c) For deeper gate than (a).

\section{EXPERIMENTAL METHOD}

We explain our device for the beam compression experiment. Fig. 1. shows a schematic diagram of the experimental arrangement. The experimental device consists of a gated electron gun which makes electron beam bunch, an induction modulator to form the compression voltage, and a solenoidal beam transport line to transport and suppress the transverse motion of the beam.

First, the electron beam bunch is formed by the electron gun. Second, a modulation (compression) voltage is applied at the acceleration gap. Finally, the beam is bunched during a bunching distance through the solenoidal transport line, in which transverse motion of the beam is suppressed by the axial field. As the front of the beam is decelerated by the modulation voltage so as to be caught up by the end of the beam, the beam bunch is compressed up to the ratio that is determined with the influence factors.

\subsection{Setup}

To make a scaled beam compression experiment in the laboratory, we used an electron beam device because the requirement for the acceleration and the modulation voltage is much lower than that of heavy ion beam [6]. The electron beam is generated by thermionic emission, and extracted as a pulsed beam by a high-speed gate pulser. We could get a beam bunch with quasi-constant energy by extracting it with electrostatic field and the gated operation of the gun.

The induction modulator is driven by five independent units as shown in Fig. 1. The output of each unit is basically sinusoidal, and their waveforms are synthesized in the modulation gap. Because high speed switching is necessary in our experiment, we used MOSFET as the switching device of each drivers.

When the beam arrive at the acceleration gap, the composite compression voltage is applied for the bunch modulation. Then, the beam is compressed during the transport trough the solenoidal line of $2 \mathrm{~m}$ in length. We drove the current of $50 \mathrm{~A}$ to the solenoid coil and a magnetic field of $0.03 \mathrm{~T}$ was formed in the transport line. The traverse motion of the beam is limited by the axial magnetic field.

\subsection{Bunch compression waveform}

When all particles in the beam bunch have the same velocity and there is no space charge effect and no collective effect, ideal compression voltage can compress the beam bunch into one point at the destination. Such ideal compression voltage waveform can be expressed in the following expressions,

$$
V(t)=\frac{m_{e}}{2 q_{e}} \cdot \frac{1}{\left(\sqrt{\frac{m_{e}}{2 q_{e} V_{0}}}+\frac{T-t}{L}\right)^{2}}-V_{0}
$$



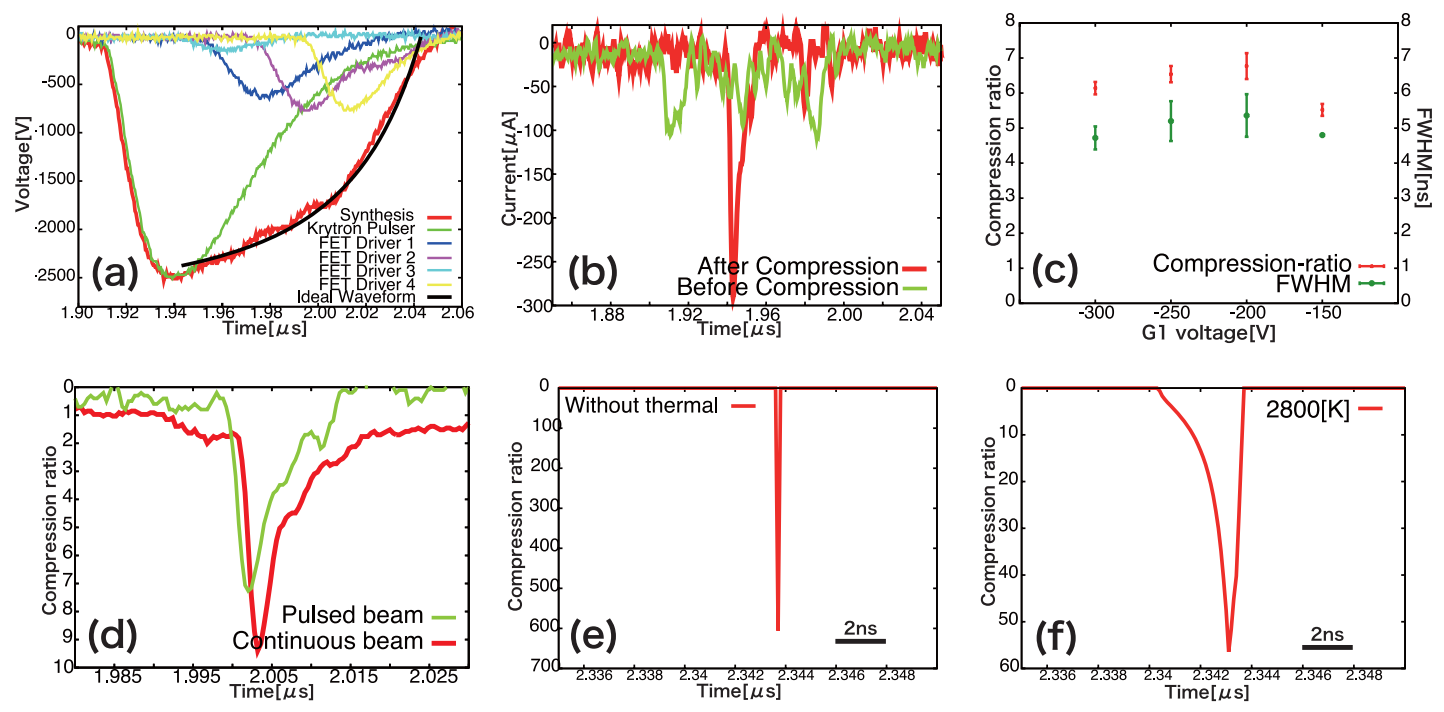

Figure 2. (a) Experimental modulation voltage waveform, (b) Beam current waveform at transport distance $\mathrm{L}=0.1 \mathrm{~m}$ and $\mathrm{L}=2 \mathrm{~m}$, (c) Compression ratio and FWHM versus G1 voltage, (d) Compression ratio of continuous beam and pulsed beam, (e) Simulation result without thermal effect, (f) Simulation result with Maxwellian distribution.

where $V_{0}$ is the extracting voltage of the beam. $\mathrm{T}$ is the pulse length of the beam and is controlled by the pulse voltage of G1. $\mathrm{L}$ is the transport length. Here $\mathrm{t}=0$ refers to the time at which the head of the beam enters the modulation gap, and the voltage applied to the tail $\mathrm{V}(\mathrm{t})$ is zero, and the voltage applied to the head is negative so that the head is decelerated. In our experiment, we compress the pulse beam of $100 \mathrm{~ns}$ with the energy of $2.8 \mathrm{keV}$ during the $2 \mathrm{~m}$ beam transport after applying the compression voltage.

\section{EXPERIMENT AND SIMULATION}

\subsection{Typical experimental result}

We show experimental modulation voltage waveform in Fig. 2(a). Synthesized waveform looks similar to ideal waveform for $100 \mathrm{~ns}$. We conducted beam compression experiment by using this waveform.

Initial beam current which is before compression is shown in Fig. 2(b). We can understand initial beam is pulsed beam with $100 \mathrm{~ns}$ length as shown in Fig. 2(b). Average current of initial beam is estimated to be $40.2[\mu \mathrm{A}]$. The beam current after compression is shown in Fig. 2(b). Peak current of final beam is estimated to be $292[\mu \mathrm{A}]$. Then the compression ratio is estimated to be 7.26. The Full Width at Half Maximum (FWHM) of the beam pulse is estimated to be $4.8 \mathrm{~ns}$ after the compression.

\subsection{Energy dispersion induced by bias voltage}

Pulsed beam is produced by the gated operation of electrode (G1). When G1 voltage is lower than cathode voltage, electrons are suppressed as shown in Fig. 1(a). When we apply pulsed voltage to G1, pulsed beam is driven to the anode region as shown in Fig. 1(b). In the case of pulsed operation, energy dispersion of electrons may occur and it may affect the compression ratio. Because there is energy difference between electrons near cathode and electrons far from cathode. Therefore, we investigated effect of G1 voltage on the compression. We measured compression ratio and FWHM versus the G1 voltage. 
Figure 2(c) shows the effect of G1 voltage on the compression ratio and FWHM. As shown, G1 voltage seems to affect the compression ratio. On the other hand, G1 voltage shows no effect on FWHM. The result indicates electrons may be lost during the transportation.

\subsection{Comparison of pulsed beam compression with continuous beam compression}

In the case of continuous beam, electrons do not have such a dispersion effect. We compared the pulsed beam compression with continuous beam compression. To generate continuous beam, we applied constant voltage on G1 electrode which is higher than cathode voltage.

Figure 2(d) is a comparison of compressed current with pulsed beam and continuous beam. Continuous beam compression ratio is estimated to be 9.38 , which is higher than that of pulsed beam (7.26). We think this result is due to the effect of energy dispersion.

\subsection{Simulation on the effect of thermal distribution}

We simulated the beam compression process using a particle transport code. At distance $\mathrm{L}=0$, we applied longitudinal velocity tilt to pulsed beam of $100 \mathrm{~ns}$. We estimated the charge flux of electrons which arrive at $\mathrm{L}=2 \mathrm{~m}$ in same time as the beam current value. In our simulation, we did not take account of space charge effect. The compressed waveform without initial energy spread is shown in Fig. 2(e). We used ideal voltage waveform for this simulation. As expected, the beam was compressed at one point in Fig. 2(e).

Electron beam ejected from the hot filament should have maxwellian velocity distribution. We simulated beam compression ratio including maxwell distribution. Electron temperature was assumed to be 2800 K. Fig. 2(f) shows simulation result which includes effect of thermal distribution. As shown in Fig. 2(f), we can estimate the effect of source temperature on the compressed waveform.

\section{CONCLUSION}

In heavy ion fusion and high energy density science using high power beams, technology to compress the beams in longitudinal direction is crucially important. We constructed a small system to study the longitudinal compression process of beams with laboratory level device.

Compression ratios were estimated as a function of initial energy spread for pulsed and continuous mode. Compression with continuous beam had higher ratio than that with pulsed beam. Effects of initial temperature was discussed numerically using a particle transport code. Result showed that the initial temperature is quite influential to beam compression. Comparisons between compression ratio and FWHM of the waveform indicates electron loss during transportation.

\section{References}

[1] A. Friedman, American Institute of Physics. 0-7354-0101-0/02 (2002)

[2] H. Qin, et al., Phys. Rev. STAB. 7, 104201 (2004)

[3] M. Reiser, JOHN WILEY \& SONS, INC. (1994)

[4] W.M. Sharp, et al., Nucl. Inst. Meth. A-544, 398 (2005)

[5] M. Tomii, T. Kikuchi, M. Nagajima, K. Horioka, 6th International IFSA Conf. (2009)

[6] R.A. Kishek, et. al., Nucl. Inst. Meth. A-544, 179-186 (2005) 